\title{
Frustrated Patients and Fearful Physicians
}

\author{
Stephen G. Henry, MD and Zachary B. Holt, MD \\ Division of General Medicine, Geriatrics, and Bioethics, University of California Davis, Sacramento, CA, USA.
}

KEY WORDS: opioid analgesics; chronic pain; patient physician relations; emotions; patient satisfaction; addiction.

$\mathrm{J}$ Gen Intern Med 32(2):148-9

DOI: $10.1007 / \mathrm{s} 11606-016-3868-y$

(C) Society of General Internal Medicine 2016

$\mathrm{M}$ aking medical treatment decisions requires an evaluation of the anticipated risks and benefits associated with available treatment options. Advocates for shared decision making emphasize the need to elicit and incorporate patients' preferences into the decision-making process. ${ }^{1}$ For many routine clinical decisions, the tradeoff between risks and benefits is relatively unambiguous (e.g., prescribing antibiotics to treat pneumonia). In these cases, discussion of treatment alternatives is usually abridged or taken for granted. After all, surveys indicate that most patients prefer to leave decision making to their physicians. $^{2}$ On the other hand, explicit discussion of risks and benefits is critical when the tradeoff between them is uncertain, or when patients and physicians are likely to disagree on the preferred treatment.

Decisions about opioids do not always fit neatly into this framework for medical decision making, because the attendant risks and benefits span multiple domains that are both difficult to compare and hard to measure. How do you weigh uncomfortable side effects against reduced pain intensity, or improved function against physical dependence? These challenges, along with the subjective nature of pain, are important reasons that decisions about opioid prescribing often frustrate both patients and physicians.

The story published by Dr. Unger in this issue of $J G I M^{3}$ makes the point that the most difficult aspects of decisions involving opioids and chronic pain are not usually related to these measurement challenges, but instead to the unpleasant emotions that arise for both patients and physicians during clinical interactions around this topic. Recognizing and managing these emotions is just as important for effective chronic pain management as is accurately assessing pain and overdose risk.

Concerns related to addiction are a major source of these negative emotions. Modern science has recognized addiction as a medical disease with biologic origins. ${ }^{4}$ Unfortunately, the general public and an alarming number of medical professionals still see addiction, either

Published online September 29, 2016 consciously or unconsciously, as a moral failing. The staff members in the story all exhibit some discomfort (manifested by their lack of engagement), and the narrator feels he is being treated like a drug addict. The story also shows the importance of nonverbal communication-a clerk's tone of voice or body language can send patients the message that they are bad people on bad medicine.

Dr. Unger's story also highlights common patient frustrations caused by recent and sudden shifts in clinical norms and guidelines around the role of opioids in chronic pain management. ${ }^{5}$ These changes are driven by new evidence that the harms from long-term opioid use are much greater than previously recognized, while the benefits remain uncertain. ${ }^{6}$ Still, it is understandable that patients feel betrayed when they are suddenly told that a medication they have been prescribed for years-and that they find effective-is now considered dangerous. This betrayal is magnified by the fear that the smallest aberrancy or vague suspicion might be cited as a reason for withholding opioids. Dr. Unger's story depicts the anger patients can feel when navigating systems many clinics have erected in the name of patient safety. Any patient might lose composure when reasonable behaviors (e.g., taking a few extra pills after twisting an ankle, switching pharmacies due to personal preference) are construed as evidence of misuse. Dr. Unger's story reminds us that these concerns leave many patients on long-term opioid therapy feeling trapped and vulnerable.

On the other hand, clinic staff members face fears of their own when it comes to opioids and pain. Only a small number of interactions with patients on long-term opioids involve overt conflict (e.g., patients shouting or threatening staff), but these leave a lasting impression and can condition staff to approach every such interaction as a potentially combative one. The secretary's limited eye contact during the opening scene probably reflects her fear of being verbally attacked rather than embarrassment over clinic policies. Physicians face additional fears, most importantly that the medications they prescribe will lead to their patients' overdose or death. These fears are compounded by the challenges of diagnosing addiction. Iatrogenic misuse and addiction do not fit neatly into the current diagnostic criteria for substance use disorders, ${ }^{7}$ leaving large gray areas that physicians must navigate when weighing the risks and benefits of continued opioid prescribing. 
In the end, making treatment decisions involving opioids for chronic pain does require weighing the risks and benefits of available treatment options. The narrator is certainly justified in expecting clinics prescribing long-term opioid therapy to arrange for routine opioid refills when the prescribing physician is on vacation. On the other hand, physicians routinely discontinue potentially life-saving medications when the risks of continuing to prescribe are too high. For example, physicians regularly stop prescribing warfarin for patients who do not comply with required lab testing and refuse to refill insulin prescriptions for patients who miss multiple appointments.

Finally, Dr. Unger's story is a well-timed reminder of our obligation as physicians to appreciate the patient's perspective and to practice empathy when treating patients suffering from chronic pain. We have a professional responsibility to help our vulnerable patients navigate the clinical uncertainties and changing landscape around opioids. Some patients may benefit from long-term, low-dose opioid therapy. Allowing these patients to suffer because of our prior traumatic experiences with a handful of angry patients is not acceptable. The goal of the clinic policies and regulations that frustrate the narrator should be to support appropriate prescribing decisions, not to punish patients or to generate pretexts for stopping opioids. When patients need to be tapered down or off opioids, physicians owe them an honest explanation that goes beyond cursory appeals to "patient safety" or "clinic policy." We must remember that we are treating people who have chronic pain, not "chronic pain patients." Our best stance in these discussions is one of compassion rather than fear.

Corresponding Author: Stephen G. Henry, MD; Division of General Medicine, Geriatrics, and Bioethics, University of California Davis, 4150 V Street, Suite 2400, Sacramento, CA 95817, USA (e-mail: sghenry@ucdavis.edu).

\section{Compliance with Ethical Standards:}

Conflict of Interest: Dr. Henry has received support through an educational grant from the ER/LA Opioid Analgesics REMS Program companies.

\section{REFERENCES}

1. Elwyn G, Frosch D, Thomson R, et al. Shared decision making: a model for clinical practice. J Gen Intern Med. 2012;27(10):1361-1367.

2. Tak HJ, Ruhnke GW, Meltzer DO. Association of patient preferences for participation in decision making with length of stay and costs among hospitalized patients. JAMA Intern Med. 2013;173(13):1195-1205.

3. Unger DN. Pain medication \& regulation: it is personal. J Gen Intern Med. 2016 (in this issue). doi: 10.1007/s11606-016-3794-z.

4. Volkow ND, McLellan AT. Opioid Abuse in Chronic Pain-Misconceptions and Mitigation Strategies. N Engl J Med. 2016;374(13):1253-1263.

5. Dowell D, Haegerich TM, Chou R. CDC guideline for prescribing opioids for chronic pain-United States, 2016. JAMA. 2016.

6. Sullivan MD, Howe CQ. Opioid therapy for chronic pain in the United States: promises and perils. Pain. 2013;154(Suppl 1):S94-100.

7. Smith SM, Dart RC, Katz NP, et al. Classification and definition of misuse, abuse, and related events in clinical trials: ACTTION systematic review and recommendations. Pain. 2013;154(11):2287-2296. 